\title{
Broncodilatadores en pacientes con bronquiolitis
}

\author{
Bronchodilators for bronchiolitis
}

Nicole Crimer

\begin{abstract}
Resumen
La bronquiolitis es una infección respiratoria aguda baja de causa viral, de aparición invernal, que es común en bebés de 0 a 12 meses de edad. Conduce a que las vías respiratorias pequeñas se inflamen y se llenen de desechos, obstruyéndose. El bebé tiene una tos fuerte, secreción nasal, generalmente fiebre y puede presentar sibilancias dificultad respiratoria y desaturación de oxígeno. Tras la presentación de un caso en la guardia se generó una controversia científica sobre la utilidad de los broncodilatadores en pacientes con bronquiolitis. Luego de realizar una búsqueda bibliográfica y seleccionar la evidencia más reciente y de mejor calidad, se concluye que la evidencia no apoya el uso de broncodilatadores en pacientes con bronquiolitis.
\end{abstract}

\section{Abstract}

Bronchiolitis is a low acute respiratory lower respiratory tract infection of viral origin, winter appearance, which is common in babies from 0 to 12 months of age. It causes the small airways in the lungs to become inflamed and fill with debris. The infant has a harsh cough, runny nose, usually fever and may have wheezing, respiratory distress and oxygen desaturation. After the presentation of a case in the emergency department, a scientific controversy was generated about the usefulness of bronchodilators in patients with bronchiolitis. After conducting a literature search and selecting the most recent and best quality evidence, it is concluded that evidence does not support the use of bronchodilators in patients with bronchiolitis.

Palabras clave: broncodilatadores, bronquiolitis, niños. Keywords: bronchodilators, bronchiolitis, children.

Crimer N.. Broncodilatadores en pacientes con bronquiolitis. Evid Actual Pract Ambul. 2019;22(1):e001077

\section{Introducción}

\section{Escenario clínico}

Un paciente de cuatro meses sin antecedentes de importancia concurrió a la Central de Emergencias Pediátricas con un síndrome de bronquiolitis. La médica interna de la guardia recomendó no usar broncodilatadores en dicha patología. En el cambio de guardia, el médico a cargo del siguiente turno, le indicó una serie con salbutamol y luego de la reevaluación refirió la mejoría del paciente generándose una controversia académica.

\section{Pregunta que generó el caso}

El uso de broncodilatadores en pacientes con diagnóstico de bronquiolitis ¿contribuye a la mejoría de los síntomas?

\section{Estrategia de la búsqueda}

Se realizó una búsqueda en PubMed utilizando los términos "bronchodilators" y "bronchiolitis" con filtros para humanos, publicaciones realizadas durante los últimos diez años, ensayos clínicos y revisiones sistemáticas. Par la confección de este artículo fueron seleccionados solamente los metaanálisis y los ensayos clínicos aleatorizados que proveyeron la evidencia de mayor calidad.

\section{Algunos datos sobre la bronquiolitis}

La bronquiolitis es un síndrome clínico que se da en niños menores de dos años y se caracteriza por la aparición de síntomas respiratorios superiores, posteriormente a la infección de las vías respiratorias inferiores, provocando su inflamación y consecuentemente, sibilancias y/o crepitantes. El principal patógeno involucrado es el Virus Sincicial Respiratorio ${ }^{1}$. La bronquiolitis se define como el primer episodio de sibilancias en un niño menor de 24 meses que tiene hallazgos físicos de infección respiratoria baja y ausencia de una explicación alternativa para las sibilancias.
Predomina durante el otoño y el invierno. La hospitalización por dicha enfermedad es la principal causa de internación de niños pequeños y tiene una incidencia máxima entre los dos y los seis meses de edad.

En cuanto a su fisiopatología, los virus infectan las células epiteliales bronquiolares terminales, causando daño directo e inflamación en los bronquios y los bronquíolos. El edema, la mucosidad excesiva y los detritus celulares desprendidos conducen a la obstrucción de las vías respiratorias pequeñas, provocando atelectasias en algunos casos. El mecanismo no involucra la broncoconstricción provocada por el músculo liso bronquial.

El síndrome está conformado por fiebre, tos y dificultad respiratoria manifestada como taquipnea, retracción, sibilancias y crepitantes. A menudo se cuenta con el precedente de congestión y/o rinorrea durante uno a tres días previos, y puede, o no, presentar desaturación de oxígeno.

Para la valoración de su gravedad y posterior evaluación en el seguimiento, existe una escala realizada por Wood Downes y modificada por FerrésTabla 1.

La bronquiolitis es generalmente una entidad autolimitada. Sin embargo, en pacientes con factores de riesgo de infección grave, o incluso sin ellos, puede requerir internación por alguno de los siguientes criterios: imposibilidad de los padres para cuidar al niño en su casa, aspecto tóxico, dificultad para la alimentación, letargo, deshidratación o dificultad respiratoria moderada a severa. Esta última se define ante la presencia de al menos una de las siguientes manifestaciones: aleteo nasal, retracciones intercostales, subcostales o supraesternales, frecuencia respiratoria mayor a 70 respiraciones por minuto, disnea o cianosis, apnea, hipoxemia con o sin hipercapnia.

Existe consenso respecto del uso de medidas de sostén junto con la suplementación de oxígeno cuando esta es necesaria, pero es controvertido el uso de broncodilatadores y esteroides.

\footnotetext{
a Servicio de Medicina Familiar y Comunitaria, Hospital Italiano de Buenos Aires. nicole.crimer@hospitalitaliano.org.ar
} 
Tabla 1. Escala Wood Downes-Ferrés, para valorar la gravedad de los pacientes con bronquiolitis.

\begin{tabular}{|c|c|c|c|c|c|c|}
\hline Puntos & Sibilancias & Tiraje & $\begin{array}{c}\text { Frecuencia } \\
\text { respiratoria } \\
\text { (VPM) }\end{array}$ & $\begin{array}{c}\text { Frecuencia } \\
\text { cardíaca (LPM) }\end{array}$ & Ventilación & Cianosis \\
\hline 0 & No & No & $<30$ & $<120$ & $\begin{array}{l}\text { Buena y } \\
\text { simétrica }\end{array}$ & No \\
\hline 1 & Fin de espiración & Subcostal & 31 a 45 & $>120$ & $\begin{array}{l}\text { Regular y } \\
\text { simétrica }\end{array}$ & $\mathrm{Si}$ \\
\hline 2 & Toda inspiración & $\begin{array}{l}\text { Subcostal e } \\
\text { intercostal }\end{array}$ & 46 a 60 & & Muy disminuida & \\
\hline 3 & Inspiración y espiración & Aleteo nasal & & & Tórax silente & \\
\hline
\end{tabular}

VPM: ventilaciones por minuto. LPM: Latidos por minuto. 1 a 3 puntos: bronquiolitis leve. 4 a 7 puntos: bronquiolitis moderada. 8 a 14 puntos: bronquiolitis grave.

\section{Resumen de la evidencia}

Se seleccionó una revisión Cochrane $^{2}$ y un ensayo clínico publicado con posterioridad a dicha revisión ${ }^{3}$.

\section{Gadomski A y col. Bronchodilators for bronchiolitis. Cochrane Database Syst Rev. 2014;(6):CD001266.}

Objetivo: evaluar los efectos de los broncodilatadores en los resultados clínicos de recién nacidos ( 0 a 12 meses) con bronquiolitis aguda.

Identificación y selección de estudios: Se realizaron búsquedas en CENTRAL MEDLINE y EMBASE hasta enero 2014 de ensayos controlados aleatorizados (ECA) comparando broncodilatadores (distintos de la epinefrina) con placebo. Dos autores seleccionaron, evaluaron la calidad de los ensayos y extrajeron los datos.

Resultados principales: fueron incluidos 30 ensayos (1.992 bebés con bronquiolitis). En 11 ECA en pacientes hospitalizados y 10 en ambulatorios, la saturación de oxígeno no mejoró con broncodilatadores ni estadística ni clínicamente $(-0,43 \%$; IC $95 \%-0,92$ a 0,06). Tampoco se redujo la tasa de hospitalización (12\% en el grupo de broncodilatadores versus $16 \%$ en el grupo de placebo (OR 0,75; IC $95 \% 0,46$ a 1,21), ni la duración de la hospitalización (Diferencia medias 0,06, IC 95\%-0,27 a 0,39). En ocho estudios de pacientes hospitalizados, no hubo cambios en el promedio del puntaje clínico con broncodilatadores. En nueve estudios ambulatorios, el puntaje clínico promedio disminuyó ligeramente con broncodilatadores, hecho que esta revisión describe como estadísticamente significativo pero de dudosa importancia clínica. Los efectos adversos incluyen taquicardia, desaturación de oxígeno y temblores.

Conclusiones de los autores: los broncodilatadores como el albuterol o el salbutamol no mejoran la saturación de oxígeno, no reducen las internaciones, no acortan la duración de la hospitalización y no reducen el tiempo de resolución de la enfermedad en el hogar. Debido a los efectos secundarios adversos y al gasto asociado con estos tratamientos, los broncodilatadores no son efectivos en el tratamiento habitual de la bronquiolitis. El metaanálisis presenta como limitación los tamaños muestrales pequeños, la falta de diseños estandarizados y la ausencia de resultados validados a lo largo de los estudios.

Flores-Gonzalez JC y col. Epinephrine Improves the Efficacy of Nebulized Hypertonic Saline in Moderate Bronchiolitis: A Randomised Clinical Trial. PLoS One, 2015. 10(11): p.e0142847.

Objetivo: evaluar la eficacia de la epinefrina (de propiedades alfa adrenérgicas) nebulizada en solución salina hipertónica al
$3 \%$ para tratar a los niños hospitalizados por bronquiolitis aguda.

Pacientes y métodos: ECA, doble ciego, controlado con placebo en 208 recién nacidos hospitalizados con bronquiolitis aguda moderada. Los bebés fueron asignados aleatoriamente para recibir solución salina hipertónica nebulizada al 3\% con $3 \mathrm{~mL}$ de epinefrina o $3 \mathrm{~mL}$ de placebo, administrados cada cuatro horas. La medida de resultado primaria fue la duración de la estadía hospitalaria.

Resultados: fueron incluidos 185 pacientes menores de 24 meses ingresados en el Hospital Universitario Puerta del Mar, Cádiz, España con diagnóstico de bronquiolitis aguda de moderada gravedad. De ellos, 94 recibieron epinefrina y 91 , placebo, entre octubre de 2011 y mayo de 2014. Fueron excluidos los pacientes con antecedentes patológicos relevantes y aquellos con diagnóstico de asma. Las características demográficas y clínicas iniciales fueron similares en ambos grupos. La duración de la estadía hospitalaria se redujo significativamente en el grupo con epinefrina en comparación con el grupo placebo $(3,94 \pm 1,88$ días frente a 4,82 $\pm 2,30$ días, $p=0,011$ ). El riesgo relativo (RR) de una estadía hospitalaria prolongada en el grupo de epinefrina fue la mitad del del grupo placebo (RR 0,45; IC95\%, 0,25 a $0,81)$. Una proporción significativamente mayor de recién nacidos que recibieron solución salina hipertónica más placebo requirió hospitalización durante más de 4 días (30,8\% vs. 13,8\% en el grupo de epinefrina; $p=0,006)$. La gravedad de la enfermedad también disminuyó significativamente más rápido en el grupo de epinefrina $(p=0,029$ y $p=0,036$ en los días 3 y 5 , respectivamente).

Conclusiones de los autores: la epinefrina nebulizada en solución salina hipertónica al $3 \%$ acorta significativamente la estadía hospitalaria en niños hospitalizados con bronquiolitis moderada aguda en comparación con solución salina hipertónica al $3 \%$ sola y mejora los puntajes clínicos de gravedad desde el tercer día de tratamiento, pero no antes.

\section{Conclusiones y recomendaciones}

Si bien el metaanálisis Cochrane concluyó que los broncodilatadores no mejoran los parámetros clínicos y aumentan los efectos adversos y los costos del tratamiento, el ECA publicado un año después, que comparó con placebo la epinefrina nebulizada, documentó un acortamiento de un día en la estadía hospitalaria y la reducción en el riesgo de una estadía prolongada.

La Sociedad Argentina de Pediatría publicó en 2014 la guía ecomendaciones para el manejo de las infecciones respiratorias agudas bajas en menores de dos años" ${ }^{4}$, donde se menciona 
que el salbutamol puede ser utilizado en el manejo inicial de la bronquiolitis debido a que induce una mejoría clínica, debido a su baja toxicidad, a su fácil administración y a la ausencia de características que permitan identificar a los niños que no responderán al tratamiento. Respalda estas recomendaciones sobre la evidencia comunicada por el metaanálisis Cochrane de 2006, que actualmente quedó desplazado por la actualización de 2014, que descarta su utilidad. La SAP desaconseja el uso de adrenalina, puesto que los beta-adrenérgicos presentan más experiencia acumulada y mayor seguridad.

Por otra parte, la Academia Americana de Pediatría, recomienda fuertemente (sobre en evidencia de nivel B) no utilizar ni epinefrina ni salbutamol en niños con diagnóstico de bronquiolitis ${ }^{5}$.

Por la evidencia identificada, considero que si bien son necesarios estudios que tomen poblaciones más grandes, el uso de broncodilatadores en la bronquiolitis no cuenta con sustento científico, aunque la epinefrina podría considerarse como una opción terapéutica.

\section{Referencias}

1. Piedra PA, Stark A. Bronchiolitis in infants and children: Clinical features and diagnosis Topic 6018 Version 40.0 . Revisado en marzo de 2018 Uptodate;Available from: http://uptodate.com.

2. Gadomski AM, Scribani MB. Bronchodilators for bronchiolitis. Cochrane Database of Systematic Reviews. 2014 jun;(6):001266. Available from: 10.1002/14651858.CD001266.pub4.

3. Flores-González JC, Matamala-Morillo MA, Rodríguez-Campoy P, Pérez-Guerrero JJ, Serrano-Moyano B, Comino-Vazquez P, et al. Epinephrine Improves the Efficacy of Nebulized Hypertonic Saline in Moderate Bronchiolitis: A Randomised Clinical Trial. PLOS ONE. 2015 nov;10(11):e0142847. Available from: 10.1371/journal.pone.0142847.

4. Moreno L, Ferrero F. SAP. Recomendaciones para el manejo de las infecciones respiratorias agudas bajas en menores de 2 años:

5. Ralston SL, Lieberthal AS, Meissner HC, Alverson BK, Baley JE, Gadomski AM, et al. Clinical practice guideline: the diagnosis, management, and prevention of bronchiolitis. Pediatrics. 2014 nov;134(5):e1474-502. Available from: 10.1542/peds.2014-2742. 